\title{
Precision of methods for calculating identity-by-descent matrices using multiple markers
}

\author{
Anders Christian Sørensen ${ }^{\mathrm{a}, \mathrm{b}, \mathrm{c} *}$, Ricardo Pong-Wong ${ }^{\mathrm{a}}$, \\ Jack J. WINDIG ${ }^{\mathrm{d}}$, John A. Woolliams ${ }^{\mathrm{a}}$ \\ ${ }^{\text {a }}$ Roslin Institute (Edinburgh), Roslin, Midlothian EH25 9PS, UK \\ b Department of Animal Breeding and Genetics, \\ Danish Institute of Agricultural Science, P.O. Box 50, 8830 Tjele, Denmark \\ ${ }^{c}$ Department of Animal Science and Animal Health, \\ Royal Veterinary and Agricultural University, Grønnegårdsvej 2, \\ 1870 Frederiksberg C, Denmark \\ d Institute for Animal Science, ID-Lelystad, P.O. Box 65, \\ 8200 AB Lelystad, The Netherlands
}

(Received 13 November 2001; accepted 22 April 2002)

\begin{abstract}
A rapid, deterministic method (DET) based on a recursive algorithm and a stochastic method based on Markov Chain Monte Carlo (MCMC) for calculating identity-by-descent (IBD) matrices conditional on multiple markers were compared using stochastic simulation. Precision was measured by the mean squared error $(M S E)$ of the relationship coefficients in predicting the true IBD relationships, relative to $M S E$ obtained from using pedigree only. Comparisons were made when varying marker density, allele numbers, allele frequencies, and the size of full-sib families. The precision of DET was $75-99 \%$ relative to MCMC, but was not simply related to the informativeness of individual loci. For situations mimicking microsatellite markers or dense SNP, the precision of DET was $\geq 95 \%$ relative to MCMC. Relative precision declined for the SNP, but not microsatellites as marker density decreased. Full-sib family size did not affect the precision. The methods were tested in interval mapping and marker assisted selection, and the performance was very largely determined by the MSE. A multi-locus information index considering the type, number, and position of markers was developed to assess precision. It showed a marked empirical relationship with the observed precision for DET and MCMC and explained the complex relationship between relative precision and the informativeness of individual loci.
\end{abstract}

IBD / genetic relationship / multiple markers / complex pedigree / information

\footnotetext{
* Correspondence and reprints

Research Centre Foulum, P.O. Box 50, DK-8830 Tjele, Denmark

E-mail: AndersC.Sorensen@agrsci.dk
} 


\section{INTRODUCTION}

The relationship between individuals has occupied researchers in genetic analysis since Fisher [9] and Wright, e.g. [28]. Their works, built upon by Henderson, e.g. [14], consider the expectation of relationship conditional on pedigree information. Except for the relationship between non-inbred parents and offspring, non-inbred monozygotic twins, and non-inbred clones, all kinds of relationships are subject to variance on the genomic level [21]. The advance of molecular genetics in recent decades have made it possible to differentiate the relationship between pairs of individuals, which according to the pedigree have the same relationship, and look deeper into the consequences [5].

Coefficients of the relationship between individuals for specific positions of the genome, i.e. genomic relationship, have been used extensively in the mapping of quantitative trait loci (QTL). In outbred populations, residual maximum likelihood (REML, [19]) is used to correct for systematic environmental factors, polygenic effects, and QTL-variances, e.g. [10]. However, this approach requires specification of a covariance structure of the QTL effect, which is the matrix consisting of the genomic relationships of individuals for a certain position of the genome. Such a matrix is also required, if breeding values are predicted using marker assisted prediction of breeding values [8].

The matrix of genomic relationships of a specific position is calculated conditional on both pedigree and marker information. This calculation is, however, not straightforward in an outbred population, when information on multiple markers is available. Simulation-based techniques, e.g. Markov Chain Monte Carlo (MCMC), present one approach to use all the marker information available. However, this method occasionally fails to converge. In these situations deterministic methods are attractive alternatives. A rapid, deterministic method for calculating the matrix using a recursive algorithm was recently presented by Pong-Wong et al. [20].

The objective of this study was to evaluate methods for calculating matrices conditional on multiple markers regarding the precision of the matrices and their performance in common animal breeding applications. Comparisons were made reflecting the different scenarios such as the density of the marker map, marker homozygosity, and population structure. In addition, an information index was developed that can be used as a simple assessment of the precision of the methods.

\section{METHODS AND MATERIALS}

\subsection{Identity-by-descent measures}

At a given locus, related individuals might have received copies of the same allele in a common ancestor. If this is the case, the alleles in the individuals are 
said to be identical by descent (IBD). The probability of this event is called the IBD probability. Likewise, if the two alleles within an individual are derived from the same ancestor they are said to be IBD. The probability of this event equals the coefficient of inbreeding of the individual.

An IBD matrix, $\mathbf{Q}$, can be defined, where the elements, $q_{(i, j)}$, are the expectation of the number of alleles carried by individual $j$ that are IBD with a randomly sampled allele from individual $i$, conditional on the genomic and pedigree information. The true IBD value, $q_{\text {true }}$, assuming full knowledge of the inheritance, is either $0,1 / 2,1$, or 2 . Consider the paternal $(p)$ and maternal $(m)$ alleles of two individuals $i$ and $j$. Then:

$$
q_{\text {true }(i, j)}=\frac{1}{2}\left(a_{p(i), p(j)}+a_{p(i), m(j)}+a_{m(i), p(j)}+a_{m(i), m(j)}\right)
$$

where $a_{x, y}$ is 1 if alleles $x$ and $y$ are IBD and 0 otherwise. Thus, the diagonal elements are either 1 or 2 , because the individual is either not inbred or completely inbred at a specific position, respectively. In the rest of this paper, IBD values refer to elements of $\mathbf{Q}$ and are, therefore, conditional expectations given pedigree and genomic information, and IBD matrix refers to $\mathbf{Q}$ unless otherwise stated.

\subsection{Calculation of IBD matrices}

When no genomic information is available, $\mathbf{Q}$ equals $\mathbf{A}$, i.e. the numerator relationship matrix [14], and this limiting form justifies the use of $\mathbf{Q}$, rather than the alternatives based on probabilities, in this study. Two methods of calculation of an IBD matrix, conditional on multiple markers, were considered in this study: a stochastic method based on MCMC techniques, and a deterministic method based on a recursive algorithm.

\subsubsection{Stochastic method}

MCMC can be used to calculate the IBD matrix conditional on multiple markers, when marker phases are not known with absolute certainty and using all available information. This method follows the procedures developed by Thompson and Heath [24], and has been implemented in the Loki software [13].

In this study, convergence was assessed for a small number of replicates for scenarios that were expected to give slow mixing of the sampler. Chains of 100000 iterations or more were run, the first 10000 were discarded, and the result was compared subjectively to the standard chain of 20000 iterations of which the first 2000 were discarded. No evidence was found to suggest that convergence had not been reached by the 20000 iterations in all the scenarios presented. Therefore, the shorter chain was used. However, evidence of lack of convergence for chains was found for biallelic markers with alleles of equal 
frequencies in populations with small full sib families and these results were not included.

A further potential problem with MCMC is the occurrence of reducible chains [7]. Reducibility of the chain occurs, if the loci have many alleles and the number of founders is small [24]. This problem was examined, following the approach explained above, when the number of alleles was larger than two, but no problems were identified.

\subsubsection{Deterministic method}

Pong-Wong et al. [20] developed a rapid method for calculating IBD matrices using multiple markers. This method partially reconstructs haplotype phases and then recursively calculates IBD values from the oldest individual to the youngest. The detailed protocol is given in [20].

This method is rapid, unlike MCMC, because it ignores markers that are not fully informative. A marker is fully informative if the phase is known in the individual and its parent, and the parent is heterozygous. The phase is established with certainty for the closest informative markers, if any, on either side of the locus. Therefore, the computationally heavy weighted summation over all possible phases, if the phase is uncertain, is avoided. On the other hand, this also means that the IBD matrix is not strictly conditional on all marker information, because not all information contained in the marker genotypes is used in the calculations. One consequence of only using subsets of the information present on the markers is that the calculated matrix is not guaranteed to be non-negative definite, unlike MCMC and exact methods. For this reason, three methods of bending $\mathbf{Q}$ to obtain a positive definite matrix were examined. The first method, denoted $\mathrm{HH}$, follows Hayes and Hill [12], and the remaining two methods, denoted $\mathrm{BB}$ and $\mathrm{BU}$, were based on changing the negative Eigenvalues. The details are given in Appendix A.

\subsection{Comparison of matrices}

\subsubsection{Direct comparison of matrices}

The matrices calculated by the MCMC and deterministic methods, respectively, were compared directly to the matrix containing the true IBD values, which was known from the simulations in this study. The criterion for comparison was the mean square error:

$$
M S E_{\text {calc }}=\frac{1}{n^{2}} \sum_{i=1}^{n} \sum_{j=1}^{n}\left(q_{\text {calc }(i, j)}-q_{\text {true }(i, j)}\right)^{2}
$$

where $n$ is the number of individuals, $q_{\text {true }}$ is the true IBD value, and $q_{\text {calc }}$ is the calculated IBD value from either MCMC, the deterministic method or from 
pedigree information. The double sum is the squared Frobenius norm of the difference of the matrices $\mathbf{Q}_{\text {calc }}$ and $\mathbf{Q}_{\text {true }}$ [6]. The Frobenius norm has been used to compare (co)variance matrices in other studies [27]. However, the $M S E$, i.e. the squared norm, was the preferred statistic in this study.

Two statistics to evaluate the methods were calculated using the MSE:

(a) The absolute efficiencies of using the marker information to obtain $\mathbf{Q}$ was calculated for the deterministic method or MCMC (subscript Det or MCMC) compared to pedigree information only (subscript $P e d$ ):

$$
\begin{aligned}
E_{A, D e t} & =\frac{\left(M S E_{P e d}-M S E_{D e t}\right)}{M S E_{P e d}} \\
E_{A, M C M C} & =\frac{\left(M S E_{P e d}-M S E_{M C M C}\right)}{M S E_{P e d}} .
\end{aligned}
$$

(b) The relative efficiency of the deterministic method compared to MCMC was calculated as follows:

$$
E_{R}=\frac{M S E_{P e d}-M S E_{D e t}}{M S E_{P e d}-M S E_{M C M C}}=\frac{E_{A, D e t}}{E_{A, M C M C}} .
$$

\subsubsection{Indirect comparison of matrices}

Whilst the MSE gives an insight into the performance of the methods, it is important to realize that the effectiveness of $\mathbf{Q}$ in applications will not be a simple function of MSE. Therefore, the matrices obtained by different methods were also compared indirectly using two applications, interval mapping and marker assisted prediction of breeding values (MAS). Other applications could have been considered as well, e.g. refining covariances among relatives for the prediction of polygenic breeding values [18], or marker assisted selection for maintaining genetic variation [26].

\section{Interval mapping}

The framework of the two-step variance component approach outlined by George et al. [10] was used for interval mapping. The first step was the calculation of the IBD matrices. The second step was REML analyses using these matrices as covariance matrices for the QTL effect. The test for a significant variance due to the QTL was performed using a likelihood ratio test $(L R)$ with a $5 \%$ significance threshold of 2.71 [23].

The analyses were only performed at position $52.5 \mathrm{cM}$. The reasons for this are that the method yields unbiased estimates of the position of a QTL, and second that previous simulations showed that the difference in test statistics for matrices obtained using MCMC and the deterministic method appears to be greatest at the position of the QTL [20]. The two methods were compared on the power to find the QTL, the size of the test statistic and the estimates of the variance components. 


\section{Marker assisted prediction of breeding values}

The second application used as an indirect comparison of the two methods of calculating the IBD matrix was MAS using the best linear unbiased prediction (BLUP) as introduced initially by Fernando and Grossman [8]. One reason for using this application is the risk of a non-positive definite matrix obtained by the deterministic method causing some predicted breeding values to go astray. The difference in predicting random effects and estimating fixed effects is that the prediction uses a regression of the differences towards zero [15]. The regression coefficient is a function of the variance estimates and the (co)variance structure and is less than one for a positive definite (co)variance matrix. However, in the case of a non-positive definite matrix the regression will regress some function of the predicted breeding values away from zero.

The variance components were assumed known and set to the simulated values, given below. The predicted QTL effects using the different IBD matrices as (co)variance structures were compared to the true QTL effects, which were known from the simulations. The correlation between the predicted and true QTL effects, i.e. the accuracy, of all animals in the pedigree was used for the comparison of the methods.

\subsection{Simulation}

\subsubsection{Population}

Two different population structures were used in this study: A population with large full-sib families, termed "pigs", and one with small full-sib families, termed "sheep". These structures offered different amounts of information for inferring phases from offspring genotypes. Both structures were simulated for four discrete generations following a non-inbred and unrelated base generation with 100 individuals born each generation making a total of 500 in the full pedigree. Selection was at random, and mating was hierarchical with random pairing of sires and dams (see Tab. I).

Table I. Details of the simulation of the two population structures called "pigs" and "sheep".

\begin{tabular}{lcc}
\hline Parameters & Pigs & Sheep \\
\hline Number of sires in each generation & 5 & 5 \\
Number of dams per sire & 2 & 10 \\
Number of male (female) offspring per mating & $5(5)$ & $1(1)$ \\
Size of paternal half-sib families & 20 & 20 \\
Size of full-sib families & 10 & 2 \\
Effective population size [2] & 14.3 & 20.0 \\
\hline
\end{tabular}




\subsubsection{Chromosomes}

One pair of chromosomes with a length of $105 \mathrm{cM}$ was simulated for each individual. Markers were simulated for each $1 \mathrm{cM}$ across the entire chromosome yielding a total of 106 markers. All animals were assumed to have known genotypes at all markers. The simulation of markers in the base population assumed linkage equilibrium, and the probability of recombination was computed using the Haldane mapping function [15]. Three subsets of the 106 markers were used in the analyses with different sizes of marker brackets:

$3 \mathrm{cM}$ : markers for each $3 \mathrm{cM}$ yielding a total of 36 markers;

$7 \mathrm{cM}$ : markers for each $7 \mathrm{cM}$ yielding a total of 16 markers;

$15 \mathrm{cM}$ : markers for each $15 \mathrm{cM}$ yielding a total of 8 markers.

Three types of markers were simulated:

2U: biallelic markers with allele frequencies 0.1 and 0.9 ;

2E: biallelic markers with allele frequency 0.5 ;

8E: markers with eight alleles with allele frequency 0.125 .

The $2 \mathrm{U}$ markers are assumed to resemble single nucleotide polymorphisms (SNP) and the 8E markers are assumed to resemble microsatellites.

At the centre of the chromosome, i.e. $52.5 \mathrm{cM}$ from each telomere, a marker with unique founder alleles was simulated in order to assess the true IBD status at that position. This actual IBD position was always in the centre of a marker bracket with a distance to the closest markers of half the size of the marker brackets. All calculations of IBD matrices were done for the position $52.5 \mathrm{cM}$.

\subsubsection{Genetic model}

For the simulation of interval mapping and MAS, phenotypes were required. The founder alleles at position $52.5 \mathrm{cM}$ were ascribed a value sampled from a normal distribution $\mathrm{N}\left(0,1 / 2 \sigma_{q}^{2}\right)$. The result of this sampling was a multiallelic, additive QTL with variance $\sigma_{q}^{2}$. See [16] for a discussion of the implications of this assumption. Also, the polygenic values, $u$, were sampled from a normal distribution $\mathrm{N}\left(0, \sigma_{a}^{2}\right)$ for the individuals of the base generation, and from a normal distribution $\mathrm{N}\left(1 / 2\left(u_{s}+u_{d}\right), 1 / 2\left(1-1 / 2\left(f_{s}+f_{d}\right)\right) \sigma_{a}^{2}\right)$ for all other individuals, where $f$ is the inbreeding coefficient [17], and the subscripts $s$ and $d$ relates to the sire and dam of the individual, respectively. A random environmental deviation was drawn from a normal distribution $\mathrm{N}\left(0, \sigma_{e}^{2}\right)$. The values of the variances used were 90,300 , and 500 for $\sigma_{q}^{2}, \sigma_{a}^{2}$, and $\sigma_{e}^{2}$, respectively. Thus, the QTL explained approx. $10 \%$ of the phenotypic variance and $23 \%$ of the genetic variance. 


\subsubsection{Simulated scenarios}

All combinations of the two population structures, three marker densities, and three levels of information content of the markers were studied, with the exception of the sheep data with biallelic markers with alleles of equal frequency (2E). This exception was because of the lack of convergence of the MCMC as implemented. This gave a total of 15 scenarios, each with 50 replicates.

The two applications, interval mapping and MAS, were used for the following four scenarios of the pig population structure:

- biallelic markers, "2E", each $3 \mathrm{cM}$;

- biallelic markers, " $2 \mathrm{E}$ ", each $15 \mathrm{cM}$;

- biallelic markers, " $2 \mathrm{U}$ ", each $3 \mathrm{cM}$;

- biallelic markers, " $2 \mathrm{U}$ ”, each $15 \mathrm{cM}$.

\subsection{Index for information from the markers}

An information index was presented in order to provide some understanding of the precision of the methods for calculating IBD matrices. It considers (a) the type of marker; i.e. the number of alleles at the marker locus and their frequencies; (b) the number of markers; and (c) the positions of the markers relative to the position of interest. The information index, $I$, attempts to quantify the precision in assessing the correct inheritance of the allele from the parent to the offspring adjusted for correct assessment by chance, i.e. when no genomic information is available. Thus, $I$ is a function of the conditional probabilities of assessing a correct inheritance pattern $(C)$ given pedigree and marker information $(M)$ and given pedigree information only $(P)$ :

$$
I=\frac{\operatorname{Pr}(C \mid M)-\operatorname{Pr}(C \mid P)}{\operatorname{Pr}(C \mid P)} .
$$

The precision using pedigree information only is the probability that an offspring inherited a specific allele from its parent, i.e. $\operatorname{Pr}(C \mid P)=\frac{1}{2}$. The adjustment in (3) is essentially the same as the correction of MSE in (1). Thus, $I$ may be considered comparable to $E_{A}$.

For an entire marker map, $\operatorname{Pr}(C \mid M)$ can be calculated, considering four possible events: (a) none of the markers are informative (NI); (b) only informative markers on the left side of the position (IL); (c) only informative markers on the right side of the position (IR); and (d) informative markers on both sides of the position $(I B)$ :

$$
\operatorname{Pr}(C \mid M)=\operatorname{Pr}(C, N I \mid M)+\operatorname{Pr}(C, I L \mid M)+\operatorname{Pr}(C, I R \mid M)+\operatorname{Pr}(C, I B \mid M) .
$$


Let $s$ be the probability of one marker being informative defined in detail later; $n_{l}$ and $n_{r}$ be the number of markers to the left and right of the position, respectively; and $r_{i}\left(r_{j}\right)$ and $r_{i j}$ be the recombination fractions between marker $i(j)$ and the position, and between marker $i$ and marker $j$, respectively, as computed from the Haldane mapping function [15]. Then the probabilities of assessing the correct inheritance pattern with the four events defined earlier are:

$$
\begin{aligned}
& \operatorname{Pr}(C, N I)=(1-s)^{\left(n_{l}+n_{r}\right)} \cdot 0.5 \\
& \operatorname{Pr}(C, I L)=(1-s)^{n_{r}} \cdot \sum_{i=1}^{n_{l}}\left((1-s)^{(i-1)} \cdot s \cdot\left(1-r_{i}\right)\right) .
\end{aligned}
$$

$\operatorname{Pr}(C, I R)$ is calculated substituting $n_{l}$ for $n_{r}$ and vice versa in the expression for $\operatorname{Pr}(C, I L)$, and

$$
\operatorname{Pr}(C, I B)=\sum_{i=1}^{n_{l}} \sum_{j=1}^{n_{r}}\left((1-s)^{(i+j-2)} \cdot s^{2} \cdot\left(1-M I N\left(r_{i}, r_{j}\right)\right)\right) .
$$

The inner bracket of (7) takes account of whether the marker information on both sides is consistent with respect to the inheritance pattern or not. Formulas (5)-(7) assume, for simplicity, that all markers have an equal probability of being informative. A more general formula, where this assumption was removed, is given in Appendix B.

The information index can be computed for both the deterministic method and MCMC. The only difference between the methods is the probability of the markers being informative, $s$, due to a difference in the use of markers, since the deterministic method only considers fully informative markers, whereas the MCMC method can use partially informative markers as long as the parent is heterozygous. The MCMC method integrates over the possible marker phases by using information from the offspring, the more offspring the more precise inferences of the phases.

\section{Probability of a marker being informative}

For the deterministic method, a marker is considered informative when it is possible to assess with certainty, which allele of an individual was inherited from the parent considered and whether that allele was the paternal or maternal allele of the parent. This occurs, when the parent is heterozygous and has a known phase, and the individual itself has a known phase. The probability of this event, $s$, is a function of the number of alleles, $m$, at the marker locus and their frequencies, $p_{1}, \ldots, p_{m}$ :

$$
s=2 \sum_{i=1}^{m-1} \sum_{j=i+1}^{m}\left(p_{i} p_{j} \cdot\left(1-p_{i} p_{j}\right)^{2}\right) .
$$


For biallelic markers with allele frequencies $p_{1}$ and $p_{2}$ (8) collapses to $s=$ $2 p_{1} p_{2}\left(1-p_{1} p_{2}\right)^{2}$. For multiallelic markers with all $m$ alleles having equal frequencies, $p=1 / m,(8)$ collapses to $s=m(m-1) \cdot p^{2} \cdot\left(1-p^{2}\right)^{2} . s$ is related to the polymorphism information content $(P I C)$ defined originally by Botstein et al. [4]. The difference between $s$ and PIC is that PIC only takes account of the parent being heterozygous and the offspring having a known phase, whereas $s$ also takes account of whether the phase in the parent is known or not.

MCMC attempts to infer unknown phases. Thus in any case where the parent is heterozygous, the marker is potentially informative. Therefore, the probability of a marker being informative, $s$, is a function of the frequency of heterozygotes and the probability of correct inference of unknown phases. This latter probability is, however, not easily calculated since it depends on the population structure. Ignoring this, the expected frequency of heterozygotes under Hardy-Weinberg equilibrium is used as $s$. This assumes that unknown phases can be inferred without error and is, therefore, an upper limit to the probability of a marker being informative for MCMC. Thus:

$$
s=1-\sum_{i=1}^{m} p_{i}^{2}
$$

where $p_{i}$ is the frequency of the $i$ th allele. $I$ can now be calculated for the deterministic method using $s$ calculated from (8) and for MCMC using $s$ calculated from (9).

Because the extra information from markers with unknown phases is not used $100 \%$ by MCMC, the ratio of the probabilities for the two methods gives a lower bound to the merit of the deterministic method relative to MCMC for a single marker at the position of interest. A plot of $s$ over a range of situations for bi- and multiallelic markers (Fig. 1) shows that $s$ increases with less variance of allele frequencies for biallelic markers and with an increasing number of alleles of multiallelic markers. However, the performance of the deterministic method relative to MCMC cannot be expected to increase monotonically with the informativeness of the markers quantified by $s$ or $P I C$, especially for biallelic markers.

\section{RESULTS}

\subsection{Direct comparison of matrices}

The average MSE for the pig population scenarios (Tab. II) and for the sheep population (results not shown) were very similar. For the average over 50 replicates, MCMC always resulted in a lower MSE than the deterministic 

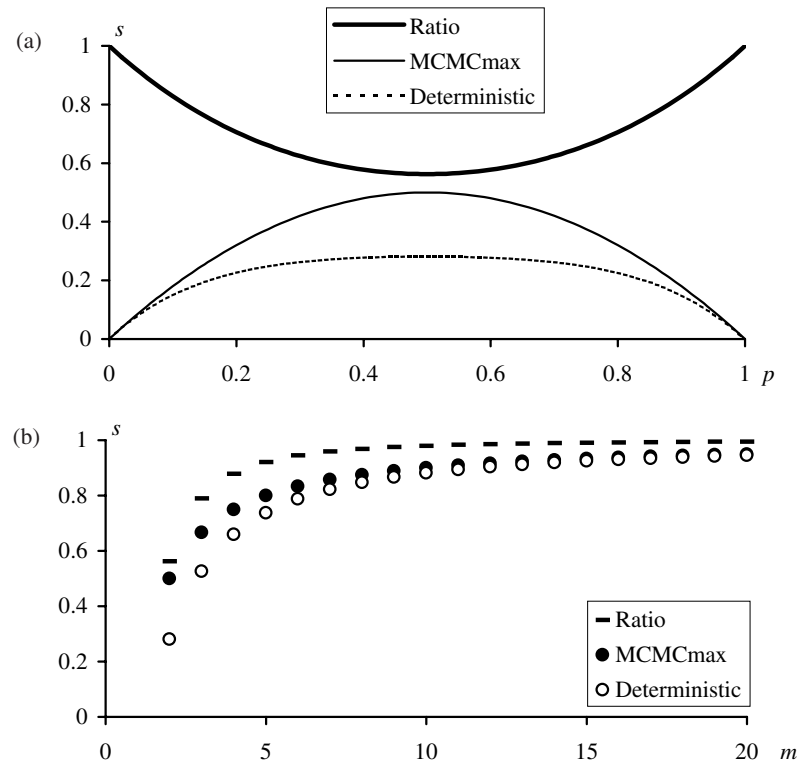

Figure 1. The probability of a marker being informative, $s$, for the deterministic method and MCMC (a) for a biallelic marker with varying allele frequency, $p$, and (b) for multiallelic markers with $m$ alleles having equal frequencies. The ratio of the probabilities for the deterministic method and MCMC is the minimum relative merit of the deterministic method when a single marker is considered.

Table II. Mean of mean square error (MSE) for the pig population of the numerator relationship matrix (Ped), MCMC, and the deterministic method (Det) versus the true IBD matrix; mean of difference (Diff) of $M S E$ of MCMC and the deterministic method; and mean of correlations of all matrix elements between true and MCMC (T-M), true and deterministic (T-D), and MCMC and deterministic (M-D).

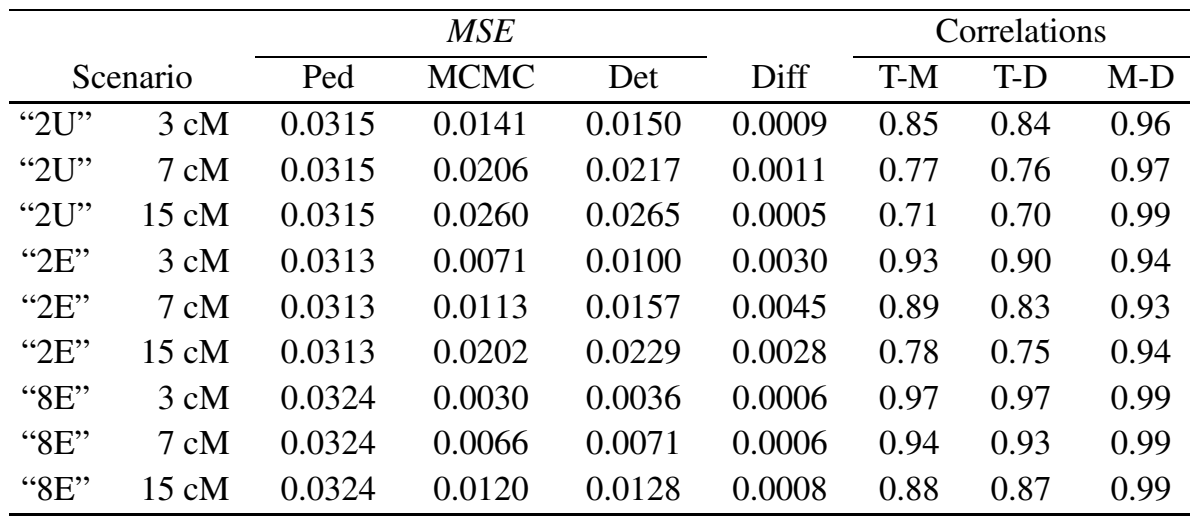

The standard errors of the means were as follows: for $M S E_{P e d}$ : 0.0006-0.0009; for $M S E_{M C M C}$ and $M S E_{D e t}$ : $0.0002-0.0006$; for Diff: $0.0001-0.0005$; and for correlations: $0.001-0.007$. 
A.C. Sørensen et al.

(a)

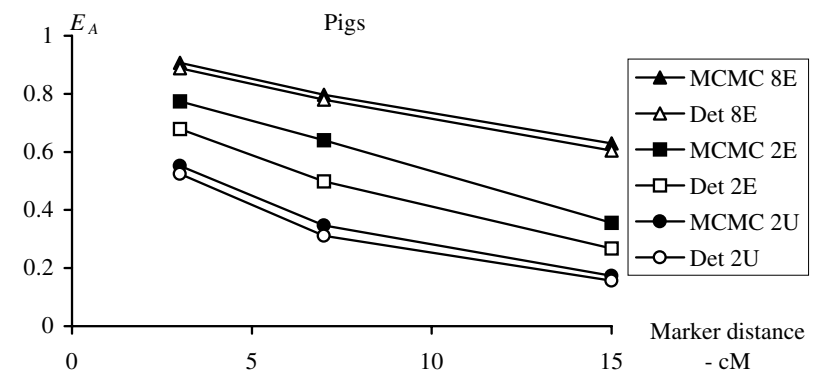

(b)

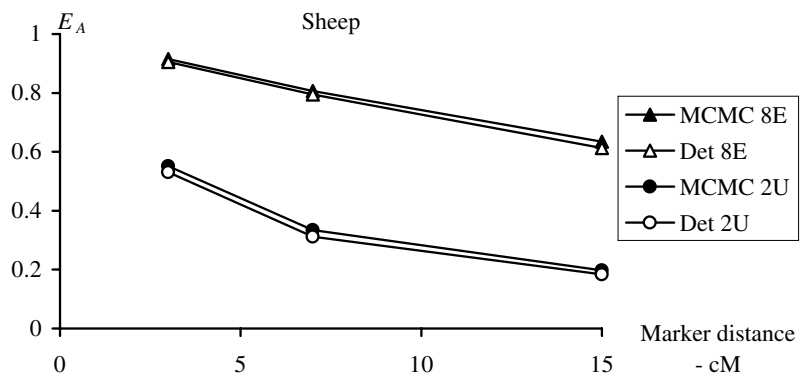

Figure 2. Absolute efficiency, $E_{A}$, calculated from (1) using MCMC and the deterministic method (Det) for the (a) pig and (b) sheep populations.

method. However, for a small number of replicates within each scenario, the deterministic method gave a smaller MSE than MCMC. As expected MSE increased when the size of the marker brackets increased. MSE increased also when the number of alleles for the markers decreased and when the frequency of heterozygotes for biallelic markers decreased. The pattern was the same when considering the entire matrix or the sub-matrix including only the last generation (results not shown). Therefore, only the results for the entire matrix are presented. This pattern was also clearly visible from the absolute efficiencies of using the marker information calculated from (1) as presented in Figure 2a for pigs and $2 b$ for sheep.

The deterministic method compared to MCMC did almost equally as well in the case of markers with eight alleles (Fig. 3). As judged by $E_{R}$, the deterministic method was only 6-10\% less efficient for biallelic markers with a skewed distribution of allele frequencies, but for biallelic markers with equal allele frequencies the deterministic method was 12-25\% less efficient. For biallelic markers, $E_{R}$ was greater for a dense marker map, e.g. $3 \mathrm{cM}$, than for a sparser map, e.g. 7 or $15 \mathrm{cM}$. The size of full-sib families seemed to have only a small impact on the relative efficiency, as the results from the pig and sheep populations agreed closely, even though there was a tendency for the relative efficiency to be higher in the case of smaller full sib families, especially for markers resembling SNP. 


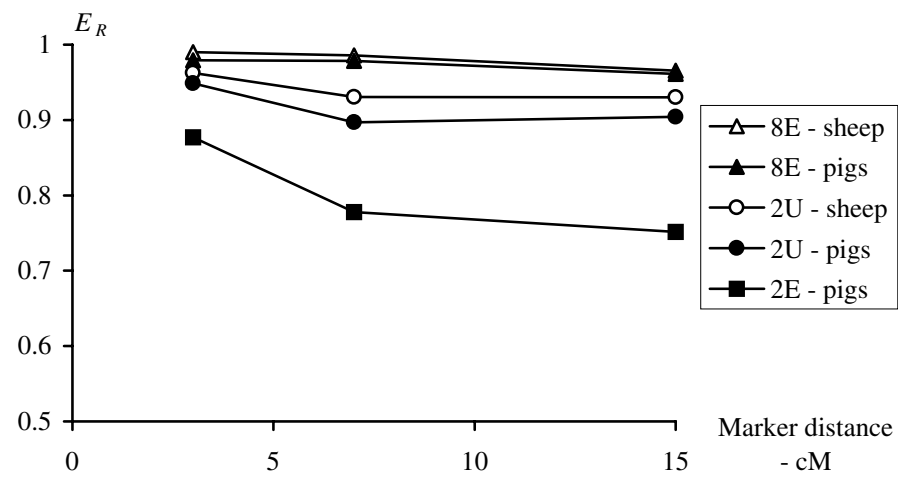

Figure 3. Relative efficiency, $E_{R}$, of the deterministic method relative to MCMC calculated from (2).

\subsection{Indirect comparison of the matrices}

In the interval mapping there was no tendency for either method to bias the average estimates of variance components (results not shown). The average test statistic increased with $E_{A}$, and so did the average accuracy of prediction of the QTL effects from MAS (Fig. 4a). However, the accuracy of prediction of the total breeding value from MAS was indifferent to the absolute efficiency, due to the limited effect of the QTL (results not shown).

The correlations of $L R$ between the two methods showed a strong relationship to $E_{R}$; but the correlations between the two methods of the accuracy of prediction of the QTL effects from MAS exhibited a weaker relationship with $E_{R}$ (Fig. 4b). One explanation for this is that the non-positive definiteness of the matrices obtained using the deterministic method could have been of greater importance in MAS than in interval mapping. The applications used in this study suggested only minor differences in the performance of the two methods, and such differences were related to $E_{R}$ as defined in (2).

The conclusion from these results was that $M S E$ on average is a good statistics for assessing the precision of matrices, especially when the matrices are to be used in interval mapping. MSE, however, does not account for the distribution and sampling of phenotypes, which, by nature affects the results from the applications.

\subsection{Eigenvalues and bending procedures}

Both the number of negative Eigenvalues of the matrices calculated using the deterministic method and their absolute sum increased with the density of the marker map, except when the markers were highly polymorphic, in which case the density did not seem to matter (results not shown). The problem was the biggest for biallelic markers with an equal allele frequency. The average 


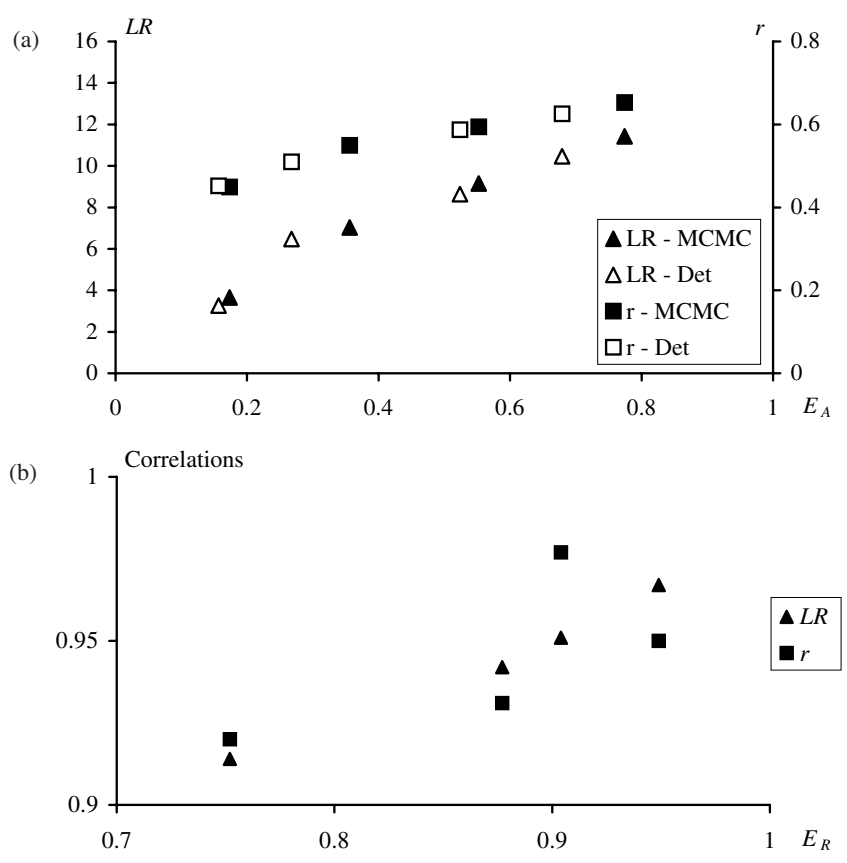

Figure 4. (a) Plot of likelihood ratio test statistics, $L R$, and accuracy of prediction of QTL effects, $r$, against the absolute efficiency, $E_{A}$, calculated from (1) for MCMC and the deterministic method (Det) for the four scenarios: (from left to right) " $2 \mathrm{U}$ " $15 \mathrm{cM}$, "2E" $15 \mathrm{cM}$, "2U" $3 \mathrm{cM}$, "2E" $3 \mathrm{cM}$. (b) Plot of correlations between MCMC and the deterministic method of the accuracy of prediction of QTL effects, $r$, and likelihood ratio test statistics, $L R$, against the relative efficiency, $E_{R}$, of the deterministic method relative to MCMC calculated from (2) for the four scenarios: (from left to right) " $2 \mathrm{E}$ " $15 \mathrm{cM}$, , $2 \mathrm{E} " 3 \mathrm{cM}$, " $2 \mathrm{U}$ " $15 \mathrm{cM}$, "2U" $3 \mathrm{cM}$.

number of negative Eigenvalues and their absolute sum were similar for the pig and sheep populations.

The effects of the three procedures of bending were similar for the pig population (Tab. III) and the sheep population (results not shown). In most cases, $\mathrm{HH}$ bending increased the MSE substantially by up to $300 \%$, compared to the original, non-positive definite matrix, and produced upwards-biased estimates of the variance due to the QTL. In addition, this bending procedure biased the regression of true QTL effects on predicted QTL effects upwards (results not shown). The two other methods of bending produced results which were very similar to each other; they reduced $M S E$ by small amounts without seriously biasing the estimates of QTL-variance or changing the size of $L R$. However, both of these procedures can result in negative off-diagonal elements of the bent matrix as well as diagonal elements less than one (results not shown). Only in a few cases did bending substantially change the predicted 
Table III. Average change in mean square error (MSE) for the pig population structure using the three methods of bending $\mathrm{HH}, \mathrm{BB}$, and $\mathrm{BU}$; average sum of the negative Eigenvalues of the matrix derived by the deterministic method (the total sum of Eigenvalues was approx. 520); and average estimate of QTL variance using the bent matrices (the simulated value was 90).

\begin{tabular}{lrrrrrrrrr}
\hline & & \multicolumn{3}{c}{ Change in $M S E, \%$} & & \multicolumn{3}{c}{ QTL variance } \\
\multicolumn{2}{c}{ Scenario } & HH & \multicolumn{1}{c}{ BB } & BU & & Sum & HH & BB & BU \\
\hline "2U" & $3 \mathrm{cM}$ & 52 & -0.26 & -0.24 & -6.9 & 250 & 90 & 91 \\
"2U" & $7 \mathrm{cM}$ & 12 & -0.05 & -0.04 & -2.0 & & & \\
"2U" & $15 \mathrm{cM}$ & 1.5 & -0.01 & -0.01 & -0.5 & 129 & 89 & 89 \\
"2E" & $3 \mathrm{cM}$ & 139 & -1.54 & -1.62 & -22.1 & 327 & 83 & 87 \\
"2E" & $7 \mathrm{cM}$ & 56 & -0.67 & -0.69 & -17.9 & & & \\
"2E" & $15 \mathrm{cM}$ & 12 & -0.12 & -0.18 & -5.3 & 223 & 99 & 100 \\
"8E" & $3 \mathrm{cM}$ & 298 & -0.25 & -0.20 & -2.4 & & & \\
"8E" & $7 \mathrm{cM}$ & 125 & -0.13 & -0.14 & -2.9 & & & \\
"8E" & $15 \mathrm{cM}$ & 48 & -0.09 & -0.05 & -2.6 & & & \\
\hline
\end{tabular}

QTL effects by regressing them towards zero. However, on average bending did not improve the accuracy of prediction.

\subsection{Relationship of $I$ and $M S E$}

For the range of scenarios, the trends and rankings of the information index, $I$, calculated from (3)-(7) using the parameters used in the simulations (Fig. 5a) were similar to the trends and rankings of $E_{A}$ (Fig. 2). However, the values of $I$ were greater than those of $E_{A}$. Parallel to this, the ratio of the information indices for the deterministic method relative to MCMC (Fig. 5b) shows trends and rankings similar to $E_{R}$ (Fig. 3).

The information index showed an empirical relationship with the natural logarithm of $E_{A}$ of the methods calculated from the simulation results (Fig. 6a). The difference between the pig and sheep populations was not significant. Contrary to the expectations, it was not possible to detect a significant difference between the deterministic method and MCMC, although the two lines in Figure 6a suggest there was a tendency for MCMC to have a higher slope as expected, because $I_{M}$ is an upper limit rather than an expectation. The empirical relationship underlines that $I$ is a good measure of the value of the information and suggests that the ratio of the expected absolute efficiencies given the relationship in Figure 6a calculated from information indices of the deterministic method $\left(I_{D}\right)$ and $\mathrm{MCMC}\left(I_{M}\right)$ can be used to predict the relative efficiency of the deterministic method using $\hat{E}_{R}=e^{3 \cdot 48 \cdot\left(I_{D}-I_{M}\right)}$. This equation 
(a)

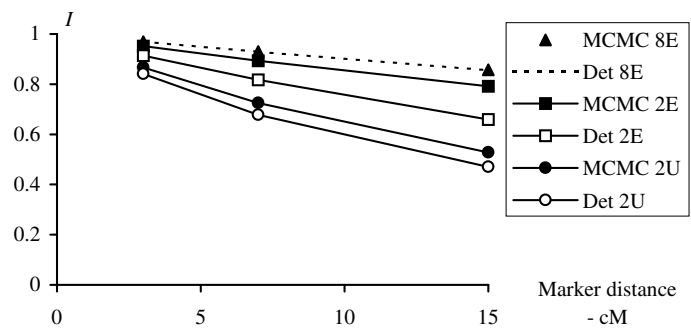

(b)

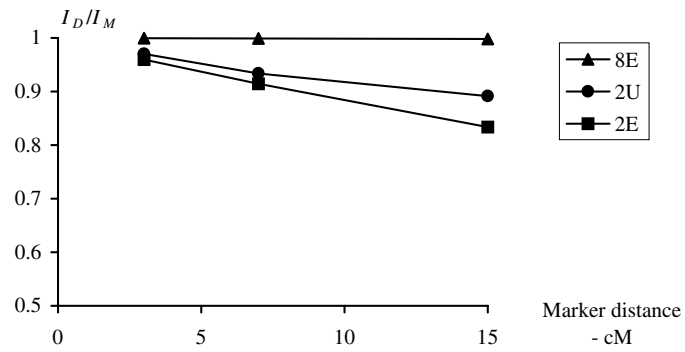

Figure 5. (a) Information indices for MCMC, $I_{M}$, and the deterministic method, $I_{D}$, and (b) ratio of information indices for the deterministic method relative to MCMC, for the scenarios used in the simulations.
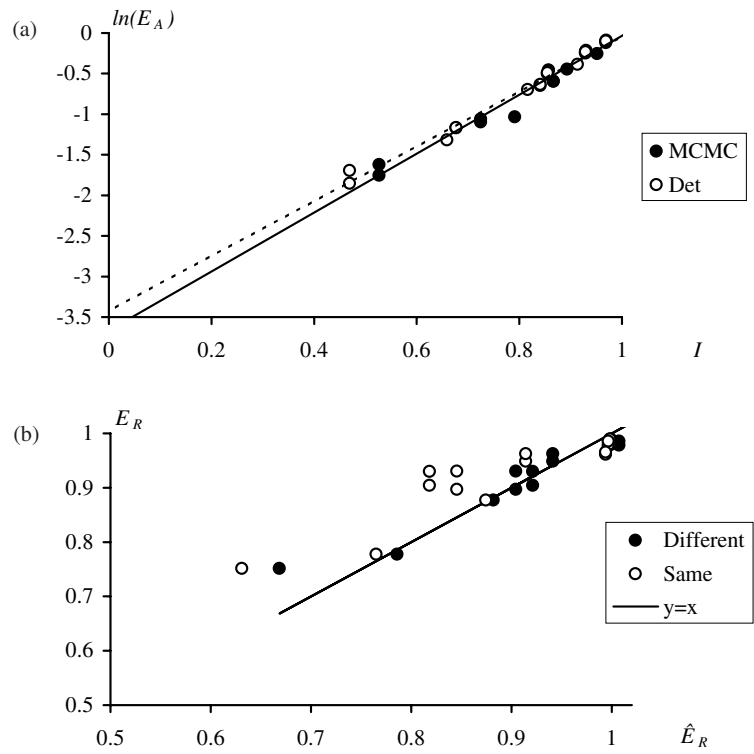

Figure 6. (a) Log-transformed absolute efficiency, $E_{A}$, for MCMC (solid trend line) and the deterministic method (dotted trend line) calculated from (1) plotted against the respective information indices, $I$, and (b) relative efficiency, $E_{R}$, plotted against the expected relative efficiency, $\hat{E}_{R}$, given the information indices of the two methods and the relationship from fitting a single line (Same) or a separate line for each method (Diff) in (a). 
was obtained by fitting a single line to all values in Figure 6a. The expression appeared to give a lower limit to $E_{R}$, except in the cases where the information indices of the two methods were very alike (Fig. 6b). When using the different lines in Figure 6 a to calculate $\hat{E}_{R}$ for the two methods, the predicted values were close to the actual values as represented by the line (Fig. 6b).

\section{DISCUSSION}

This study has presented the results of a comparison of a deterministic method and an MCMC based method for calculating IBD matrices for a number of scenarios of population structure, density of marker map, and heterozygosity of markers. It was shown that the deterministic method ranges in efficiency from 75 to $99 \%$ as judged by the MSE. The MSE determined very largely the effectiveness of the different methods for calculating IBD matrices for interval mapping and MAS. The marker type and spacing could be used to derive an information index that provides a good ranking of alternatives in terms of the information provided by the markers.

The precision of the deterministic method relative to MCMC is a complex function of the amount of marker information available. This is evident from the reranking of scenarios going from absolute (Fig. 2) to relative efficiencies (Fig. 3), which is closely related to the probability of the methods finding informative markers. For multiallelic markers, the relative merit of the deterministic method increases with the amount of information, i.e. the number of the alleles (Fig. 1b). However, for biallelic markers, the relative merit of the deterministic method decreases with increasing amounts of information, i.e. the frequency of heterozygotes (Fig. 1a). This occurs because with an increasing difference of allele frequencies there is less information with which MCMC can work that is not available to the deterministic method. Thus, one cannot generalise from the amount of information, e.g. as judged by PIC [4], to the efficiency of deterministic methods relative to MCMC. Based on the simulations, the precision of the deterministic method is very close to MCMC for multiallelic markers resembling microsatellites and for SNP in outbred populations, where alleles are of unequal frequencies, e.g. [3], whereas the efficiency of deterministic methods relative to MCMC is expected to be less in crosses of inbred lines, where allele frequencies are close to 0.5 .

The $M S E$, and $E_{A}$ derived from it, provided a good representation of the performance of the different methodologies in practical applications. $E_{A}$ was initially chosen because of its computational simplicity, but its use as a basis of comparison was tested by examining the outcome from using the derived matrices for interval mapping, e.g. [20] and MAS, e.g. [16]. The outcome showed that in both cases the performance as judged by the criteria ( $L R$ in 
interval mapping and accuracy of prediction in MAS) was closely related to $E_{A}$ (Fig. 4a). This justified the use of $E_{A}$ as a reasonable criterion for comparison.

The precision of the realised matrices from (1) and the expected precision calculated from the multi-locus information index, (3)-(7), corresponded well since the ranking of the scenarios was very highly correlated. This relationship is even clearer from Figure 6a, which indicates a strong empirical relationship that suggests its use in predicting the absolute and relative efficiencies. The ability to infer relative efficiencies is due to the informativeness of each single marker calculated given the method, i.e. deterministic or MCMC. Thus, in situations where simulations are not possible, the information index can be used as a guideline in choosing to use the deterministic method or MCMC, or simply to assess the expected efficiency of the method used given the array of markers and their properties. The empirical relationship is non-linear, because $I$ only considers the IBD status between the parent and offspring, whereas $E_{A}$ is calculated from matrices containing IBD values for all kinds of relationships.

The index will have limitations mainly to do with the size and structure of the population. One possibility that was explored was the full-sib family size, but this had little impact. Nevertheless, we believe that population attributes such as mating structure, e.g. systematic deviations from the Hardy-Weinberg equilibrium, or the particular subset of individuals being predicted, e.g. close to the base generation or many generations from it, will influence the observed $M S E$. However, we believe the index will still provide a useful ranking of options related to markers and methods albeit population specific.

Missing marker genotypes might present another limitation to the information index. The comparison of methods in this study was performed assuming perfect knowledge of all marker genotypes of all individuals in the pedigree. However, the methods handle situations where marker genotypes are missing in different ways: MCMC integrates over all possible genotypes, whilst the deterministic method treats the unknown marker genotypes as uninformative. Due to this difference, the relative efficiency of the deterministic method is expected to decrease relative to MCMC with increasing frequency of missing marker genotypes. The expected absolute efficiency for the deterministic method can be calculated from the index for cases where genotypes are missing randomly over animals and loci. However, when animals or entire generations are not genotyped the performance of the deterministic method is not easily assessed. Future research might direct attention to how much missing marker information is tolerable in order for the deterministic method to still perform satisfactorily. Because the properties of deterministic methods in situations with missing markers have not yet been explored, MCMC is the method of choice in such cases.

The deterministic method used in this study is not guaranteed to produce positive semi-definite matrices. This appears to be a result of calculating IBD 
for sibs in a pair-wise fashion [20]. The size of this problem, as measured by the number of negative Eigenvalues, is partly related to the amount of marker information. The calculated IBD matrix, $\mathbf{Q}_{\text {calc }}$, has two limiting forms, $\mathbf{A}$ and $\mathbf{Q}_{\text {true }}$, which are approached as the marker information becomes very limited or very accurate, respectively. As $\mathbf{Q}_{\text {calc }}$ approaches either of these limiting forms, as judged by $E_{A}$, the number of negative Eigenvalues decreases, because $\mathbf{A}$ is positive definite and $\mathbf{Q}_{\text {true }}$ is positive semi-definite. However, when $\mathbf{Q}_{\text {calc }}$ is at a distance from both limiting forms, the number of negative Eigenvalues could be very high.

Three methods of bending were examined in this study of which BU bending is the method of choice among those considered here in situations where a positive definite matrix is indispensable, especially in MAS. The HH method of bending was originally designed for smaller matrices in multi-trait analyses, and in this study, in a different context, it did not perform satisfactorily, since it inflated the MSE substantially and resulted in upwards-biased estimates of the QTL-variance. One explanation may be the confounding with the polygenic effect caused by bending it towards the numerator relationship matrix. In contrast, the matrices bent using $\mathrm{BB}$ or $\mathrm{BU}$, performed very similar to the unbent matrices, and had the added property of being positive definite. BB bending, which biases the sum of the Eigenvalues upwards, biases the estimate of the average coefficient of inbreeding, since the sum of the Eigenvalues equals the trace of the matrix [22], and their average is equal to one plus the average coefficient of inbreeding in the population. This bias does not occur with BU.

MCMC is a powerful tool to use all available information when calculating IBD matrices in complex pedigrees. However, for very tight linkage, e.g. with very dense marker maps, the mixing properties of MCMC deteriorate [24]. In addition, convergence of MCMC is difficult to diagnose. The deterministic method can be used as an alternative when convergence of MCMC cannot be achieved, and the results of this study suggest that the loss of precision, in effect, from using deterministic methods is limited in situations with a dense marker map of SNP, especially if these have rare alleles, and in situations with very polymorphic microsatellite markers in both dense and sparse marker maps. Additionally, this paper presents an index, which can be a useful tool in assessing the information content of a data set without using simulations and may, therefore, play a role in evaluating the impact of marker assisted selection or the power of linkage disequilibrium studies.

\section{ACKNOWLEDGEMENTS}

The authors gratefully acknowledge funding from the Royal Veterinary and Agricultural University, Copenhagen, Denmark; the Biotechnology and Biological Sciences Research Council (BBSRC), UK; SENTER (BTS-project) 
and Holland Genetics; and the Department for Environment, Food, and Rural Affairs (DEFRA), UK. We would also like to thank Dr. S.C. Heath for generously allowing us the use of his Loki software, and Dr. A.W. George for useful comments on using Loki.

\section{REFERENCES}

[1] Anonymous, NAG Fortran Library Introductory Guide Mark 16, 1st ed., NAG Ltd, Oxford, 1993.

[2] Bijma P., Van Arendonk J.A.M., Woolliams J.A., A general procedure to predict rates of inbreeding in populations undergoing mass selection, Genetics 154 (2000) 1865-1877.

[3] Blott S.C., Williams J.L., Haley C.S., Genetic relationships among European cattle breeds, Anim. Genet. 29 (1998) 273-282.

[4] Botstein D., White R.L., Skolnick M., Davis R.W., Construction of a genetic linkage map in man using restriction fragment length polymorphisms, Am. J. Hum. Genet. 32 (1980) 314-331.

[5] Christensen K., Fredholm M., Winterø A.K., Jørgensen J.N., Andersen S., Joint effect of 21 marker loci and effect of realized inbreeding on growth in pigs, Anim. Sci. 62 (1996) 541-546.

[6] Duff I.S., Erisman A.M., Reid J.K., Direct methods for sparse matrices, 1st ed., Clarendon Press, Oxford, 1986.

[7] Feller W., An introduction to probability theory and its applications, 1st ed., John Wiley \& Sons, New York, 1968.

[8] Fernando R.L., Grossman M., Marker assisted selection using best linear unbiased prediction, Genet. Sel. Evol. 21 (1989) 467-477.

[9] Fisher R.A., The correlation between relatives on the supposition of mendelian inheritance, Trans. Roy Soc. Edin. 52 (1918) 399-433.

[10] George A.W., Visscher P.M., Haley C.S., Mapping quantitative trait loci in complex pedigrees: a two-step variance component approach, Genetics 156 (2000) 2081-2092.

[11] Gunawan B., James J.W., The use of "bending" in multiple trait selection of Border Leicester-Merino synthetic populations, Aust. J. Agric. Res. 37 (1986) 539-547.

[12] Hayes J.F., Hill W.G., Modification of estimates of parameters in the construction of genetic selection indices ("bending"), Biometrics 37 (1981) 483-493.

[13] Heath S., Markov chain Monte Carlo segregation and linkage analysis for oligogenic models, Am. J. Hum. Genet. 61 (1997) 748-760.

[14] Henderson C.R., A simple method for computing the inverse of a numerator relationship matrix used in the prediction of breeding values, Biometrics 32 (1976) 69-83.

[15] Lynch M., Walsh B., Genetics and analysis of quantitative traits, 1st ed., Sinauer Associates, Sunderland, 1998.

[16] Meuwissen T.H.E., Goddard M.E., The use of marker haplotypes in animal breeding schemes, Genet. Sel. Evol. 28 (1996) 161-176. 
[17] Meuwissen T.H.E., Luo Z., Computing inbreeding coefficients in large populations, Genet. Sel. Evol. 24 (1992) 305-313.

[18] Nejati-Javaremi A., Smith C., Gibson J.P., Effect of total allelic relationship on accuracy of evaluation and response to selection, J. Anim. Sci. 75 (1997) 1738-1745.

[19] Patterson H.D., Thompson R., Recovery of inter-block information when block sizes are unequal, Biometrika 58 (1971) 545-554.

[20] Pong-Wong R., George A.W., Woolliams J.A., Haley C.S., A simple and rapid method for calculating identity-by-descent matrices using multiple markers, Genet. Sel. Evol. 33 (2001) 453-471.

[21] Rasmuson M., Variation in genetic identity within kinships, Heredity 70 (1993) 266-268.

[22] Searle S.R., Matrix algebra useful for statistics, 1st ed., John Wiley \& Sons, New York, 1982.

[23] Self S.G., Liang K.-Y., Asymptotic properties of maximum likelihood estimators and likelihood ratio tests under non-standard conditions, J. Am. Stat. Assoc. 82 (1987) 605-610.

[24] Thompson E.A., Heath S.C., Estimation of conditional multilocus gene identity among relatives, in: Seillier-Moiseiwitsch F. (Ed.), Statistics in Molecular Biology and Genetics, Institute of Mathematical Statistics, New York, Lecture Notes-Monograph Series, Vol. 33, 1999, pp. 95-113.

[25] Thompson R., Atkins K.D., Sources of information for estimating heritability from selection experiments, Genet. Res. 63 (1994) 49-55.

[26] Toro M., Silió L., Rodrigánez J., Rodriguez C., Fernández J., Optimal use of genetic markers in conservation programmes, Genet. Sel. Evol. 31 (1999) 255261.

[27] Vonesh E.F., Chinchilli V.M., Pu K., Goodness-of-fit in generalized nonlinear mixed-effects models, Biometrics 52 (1996) 572-587.

[28] Wright S., Coefficients of inbreeding and relationship, Am. Nat. 56 (1922) 330338.

\section{APPENDIX A: BENDING OF NON-POSITIVE DEFINITE MATRICES}

The Eigenvalues and Eigenvectors of $\mathbf{Q}$ were computed using a NAG subroutine [1] in order to assess the definiteness of the matrices calculated using MCMC and the deterministic method.

For an IBD matrix to be consistent with its use as a (co)variance matrix it must be positive definite, or at least positive semidefinite, although the matrix is not invertable in this case. A positive definite matrix has Eigenvalues, which are all greater than 0 [22]. A matrix with some positive and some negative Eigenvalues is non-positive definite. The problem of negative Eigenvalues has been encountered e.g. in genetic parameter estimation, and in this context Hayes and Hill [12] described a procedure called bending by which a positive definite 
matrix can be derived from a non-positive definite matrix. Bending changes the distribution of Eigenvalues, which in the case of a relationship matrix holds information of the population structure [25]. Thus, any inconsistencies of elements of the matrix are eliminated. In this study, three different types of bending were assessed for the efficiency of deriving a positive definite matrix without seriously changing the matrix.

The $\mathrm{HH}$ method was originally proposed for an estimated genetic (co)variance matrix of traits to be used in multi-trait selection index calculations [12]. They proposed to change the matrix in the direction of a positive definite matrix with an appropriate structure. In the case of an IBD matrix, an appropriate structure could be the additive genetic relationship matrix, A [14]. The bent matrix, $\mathbf{Q}^{*}$, of $\mathbf{Q}$ towards $\mathbf{A}$ was computed as follows:

$$
\mathbf{Q}^{*}=(1-\gamma) \mathbf{Q}+\gamma \lambda \mathbf{A}
$$

where $\lambda$ is the mean of the Eigenvalues of $\mathbf{Q}$, and $\gamma$ is the bending factor, which should be big enough to make the smallest Eigenvalue of $\mathbf{Q}$ slightly bigger than zero. The size of the bending factor is related to the absolute value of the smallest Eigenvalue [11]. $\mathbf{Q}$ is undergoing bigger modifications, the bigger the absolute value of the smallest Eigenvalue. This procedure was referred to as the Hayes \& Hill bending.

The second and third method of bending directly modifies the Eigenvalues of $\mathbf{Q}$. The negative Eigenvalues were changed to a small positive value in both methods. The BB method leaves the positive Eigenvalues unmodified thereby biasing their sum upwards, and correspondingly biasing the mean inbreeding coefficient. The BU method modifies all the positive Eigenvalues by regressing them by an equal proportion towards zero in order to keep the sum of the Eigenvalues unbiased. The bent matrix, $\mathbf{Q}^{*}$, was computed from the modified Eigenvalues and the original Eigenvectors as follows:

$$
\mathbf{Q}^{*}=\mathbf{U D}^{*} \mathbf{U}^{\prime}
$$

where $\mathbf{U}$ is a matrix with the columns being the Eigenvectors of $\mathbf{Q}$, and $\mathbf{D}^{*}$ is a diagonal matrix with the modified Eigenvalues on the diagonal.

\section{APPENDIX B: GENERALIZATION OF THE INFORMATION INDEX}

The information index (3) and (4) can be calculated in situations where the markers have different probabilities of being informative, $s_{i}$, using (B.1)-(B.3) 
instead of (5)-(7):

$$
\begin{aligned}
& \operatorname{Pr}(C, N I)=\left(\prod_{i=1}^{n_{l}+n_{r}}\left(1-s_{i}\right)\right) \cdot 0.5 \\
& \operatorname{Pr}(C, I L)=\left(\left(\prod_{i=1}^{n_{r}}\left(1-s_{i}\right)\right) \cdot \sum_{i=1}^{n_{l}}\left(\left(\prod_{j=1}^{i-1}\left(1-s_{j}\right)\right) \cdot s_{i} \cdot\left(1-r_{i}\right)\right)\right) .
\end{aligned}
$$

$\operatorname{Pr}(C, I R)$ is calculated substituting $n_{l}$ for $n_{r}$ and vice versa in $\operatorname{Pr}(C, I L)$, and $\operatorname{Pr}(C, I B)$

$$
=\sum_{i=1}^{n_{l}} \sum_{j=1}^{n_{r}}\left(\left(\prod_{k=1}^{i-1}\left(1-s_{k}\right)\right) \cdot s_{i} \cdot\left(\prod_{l=1}^{j-1}\left(1-s_{l}\right)\right) \cdot s_{j} \cdot\left(1-\operatorname{MIN}\left(r_{i}, r_{j}\right)\right)\right) .
$$

